\title{
Determining the Significant Part in an Electrical appliance using Fuzzy Cognitive Map and to Minimize the Cost in the Creation of a Circuit
}

\author{
A. Kanchana, Vinitha Navis Varuvel, D. Samundeeswari, S. Kuppuraj, R. Kiruthika
}

\begin{abstract}
There are many parts in an electrical appliance, each performing various operations. In particular, if a part fails, then the appliance will not be able to carry out its operations in a better way. Thus, to determine the significant part, fuzzy cognitive map can be used. However, it is noticed that in the washing machine, the outcome of the experiment lists all the parts to play an equal role. Therefore, in order to reduce the cost, the circuit must be minimized which can be done using a Java script software. Using the above circuit in the washing machine, it is easy to identify the fault in the part even before it gets damaged.

Keywords: Washing Machine, Electrical parts, Fuzzy cognitive map, Java script software.
\end{abstract}

\section{INTRODUCTION}

The concept of Boolean function was introduced by George Boole in 1854. In 1938[1], Claude Shannon designed switching circuits based on Boole's concept which is applied in the field of Computer Science.

Many methods were employed in this regard which is also explained in the papers [2] and [3]. Even though these methods did not reduce the variables, we can use the circuit in some problems similar to the ones solved in paper [4]. In such a case, how can the cost be reduced? In order to reduce the cost of the circuit, the number of variables should be minimized in the Boolean function. Paper [5] gives the solution for the above problem by using the technique of Fuzzy Cognitive Map [6] for any number of variables illustrated using a refrigerator. The drawback of the above process is that it does not hold for all the electrical appliances. This can be explained with the example of Washing machine. In this paper, we notice that in a washing machine all the variables have equal roles to play.

Revised Manuscript Received on December 30, 2019.

* Correspondence Author

A. Kanchana, Assistant Professor of Mathematics, Rajalakshmi Institute of Technology, Kuttambkkam, Chennai. Kanchana. anbazhagan@gmail.com

Vinitha Navis Varuvel, Assistant Professor of Mathematics, Rajalakshmi Institute of Technology, Kuttambkkam, Chennai. Kanchana. anbazhagan@gmail.com

D. Samundeeswari, Assistant Professor of Mathematics, Rajalakshmi Institute of Technology, Kuttambkkam, Chennai. Kanchana. anbazhagan@gmail.com

S. Kuppuraj, Assistant Professor of Mathematics, Rajalakshmi Institute of Technology, Kuttambkkam, Chennai. Kanchana. anbazhagan@gmail.com

R. Kiruthika, Assistant Professor of Mathematics, Rajalakshmi Institute of Technology, Kuttambkkam, Chennai. Kanchana. anbazhagan@gmail.com

(C) The Authors. Published by Blue Eyes Intelligence Engineering and Sciences Publication (BEIESP). This is an open access article under the CC BY-NC-ND license (http://creativecommons.org/licenses/by-nc-nd/4.0/)
So, once again the question is raised as to how to minimize the cost in the creation of a circuit connecting all the parts as in paper [4]. This is done using the Java script software as in paper [7] which is explained below.

\section{MATERIAL AND METHODS}

\section{A. Fuzzy Cognitive Map}

Networks of complex system can be solved by Fuzzy Cognitive Map [8] which was introduced by Bart Kosko. A fuzzy relational matrix is created with the help of the equation given by,

$R_{i j}=\left(r_{i j}\right)$,

where $r_{i j}$ stands for the impact of one variable on another with membership values.

\section{B. Java Scripts}

Java Script is an object-oriented scripting language which can be connected to the objects of its environment to provide programmatic control over them. Core JavaScript can be extended for a variety of purposes by supplementing it with additional objects. The JavaScript language resembles Java but does not have Java's static typing and strong type checking. JavaScript follows most Java expression syntax, naming conventions and basic controlflow constructs.

\section{FUZZY COGNITIVE MAP}

The problem is considered with an application of washing machine. By taking eleven important parts as variables, the problem is modeled as follows:

\section{MODELING OF THE PROBLEM}

The algorithm of Fuzzy Cognitive Map model is designed in such a way that the variables have both positive as well as negative impact on each other. Here, the motive of the work is "Good Working Condition of Washing Machine", which is taken as one of the variable.

Also, the Linguistic term with membership values is to be fixed. Since the parts of the washing machine have positive impacts on its working condition, it is always fixed in a positive mode.

Table-I: (Membership Values)

\begin{tabular}{|c|c|}
\hline Linguistic Term & Membership Value \\
\hline Very High(VH) & 100 \\
\hline High(H) & 80 \\
\hline Medium(M) & 60 \\
\hline Low(L) & 40 \\
\hline Very Low(VL) & 20 \\
\hline
\end{tabular}




\section{Determining the Significant Part in an Electrical appliance using Fuzzy Cognitive Map and to minimize the cost in the Creation of a Circuit}

From Table I, it is clear that the values are represented in the interval $[0,100]$ for the purpose of the software (Java Programmed applet), but it is usually represented in the interval $[0,1]$.

Now, the problem is framed by defining the variables as given below:

- $\mathrm{V}_{1}$ - Water Pump

- $\mathrm{V}_{2}$ - Water inlet control valve.

- $\mathrm{V}_{3}$ - Drum.

- $\mathrm{V}_{4}$ - Agitator or paddles.

- $\quad \mathrm{V}_{5}-$ Washing machine motor.

- $\mathrm{V}_{6}$ - Drain pipe.

- $\quad \mathrm{V}_{7-}$ Printed circuit board (PCB).

- $\mathrm{V}_{8}$ - Timer.

- $\mathrm{V}_{9}$ - Heating element.

- $\mathrm{V}_{10^{-}}$Good working condition of washing machine.

V1. Water Pump:

It is used for circulating the water through the wash cycle and also for draining the water during the spin cycle.

$V_{2}$. Water inlet control valve:

It is located near the water inlet point. When we load the clothes, depending on the amount of water required, it opens and closes automatically.

\section{Drum:}

There are two drums. The inner drum is perforated with holes to allow the water in and out. Clothes are loaded in the inner drum, which moves around the washing machine. The outer drum supports the inner drum. It contains the inner drum and the water, stopping it from leaking into the rest of the machine.

\section{V4. Agitator or paddles:}

This is located inside the tub of the washing machine and helps perform the cleaning of the clothes. A fullyautomatic washing machine has these paddles on the inner drum which is controlled by a rotating disc. A semiautomatic washing uses an agitator that rotates within the machine to produce a current in the machine. These are designed to move the clothes around the wash to allow the detergent to work and remove dirt particles and soiling from the clothes, helping the clothes rub together while washing.

\section{$V_{5}$. Washing machine motor:}

It is combined with the agitator or the disc that turns the drum and produces a rotator motion. This mechanism keeps the machine going.

\section{V6. Drain pipe:}

The dirty water from washing is expelled from the machine via the drain pipe.

\section{V7. Printed circuit board (PCB):}

The electronics that operate the machine, from electrical components to circuits, is found here. It can be programmed and help operate the machine, acting as the artificial intelligence for the washing machine. Sometimes, it also decides the time needed for rinsing or washing.

\section{V8. Timer:}

It helps to set the wash time for the clothes, either manually or automatically.

\section{Heating element:}

It heats the water in the washing machine to the desired temperature.
$V_{10}$ - Good working condition of washing machine:

This variable is very important which in turn is affected by all other variables.

Table II: The relational matrix

\begin{tabular}{|c|c|c|c|c|c|c|c|c|c|c|}
\hline $\begin{array}{c}\text { Impact } \\
\text { Values }\end{array}$ & $\mathrm{V}_{1}$ & $\mathrm{~V}_{2}$ & $\mathrm{~V}_{3}$ & $\mathrm{~V}_{4}$ & $\mathrm{~V}_{5}$ & $\mathrm{~V}_{6}$ & $\mathrm{~V}_{7}$ & $\mathrm{~V}_{8}$ & $\mathrm{~V}_{9}$ & $\mathrm{~V}_{10}$ \\
\hline $\mathrm{V}_{1}$ & - & $\mathrm{M}$ & $\mathrm{M}$ & - & - & $\mathrm{VH}$ & - & - & $\mathrm{L}$ & $\mathrm{H}$ \\
\hline $\mathrm{V}_{2}$ & $\mathrm{H}$ & - & $\mathrm{VH}$ & - & - & - & - & - & - & $\mathrm{M}$ \\
\hline $\mathrm{V}_{3}$ & $\mathrm{M}$ & $\mathrm{M}$ & - & - & $\mathrm{L}$ & - & - & - & $\mathrm{H}$ & $\mathrm{M}$ \\
\hline $\mathrm{V}_{4}$ & - & - & $\mathrm{VH}$ & - & $\mathrm{H}$ & - & - & $\mathrm{L}$ & $\mathrm{VH}$ & $\mathrm{VH}$ \\
\hline $\mathrm{V}_{5}$ & - & - & $\mathrm{VH}$ & $\mathrm{VH}$ & - & - & - & - & - & $\mathrm{VH}$ \\
\hline $\mathrm{V}_{6}$ & $\mathrm{H}$ & $\mathrm{VH}$ & $\mathrm{H}$ & $\mathrm{M}$ & - & - & - & - & - & $\mathrm{M}$ \\
\hline $\mathrm{V}_{7}$ & - & - & - & $\mathrm{H}$ & $\mathrm{VH}$ & - & - & $\mathrm{VH}$ & - & $\mathrm{VH}$ \\
\hline $\mathrm{V}_{8}$ & - & - & - & $\mathrm{M}$ & $\mathrm{M}$ & - & $\mathrm{VH}$ & - & - & $\mathrm{M}$ \\
\hline $\mathrm{V}_{9}$ & - & - & $\mathrm{VH}$ & - & $\mathrm{M}$ & - & - & - & - & $\mathrm{L}$ \\
\hline $\mathrm{V}_{10}$ & - & - & - & - & - & - & - & - & - & - \\
\hline
\end{tabular}

Table II describes the level of efficiency of one part with respect to the other parts. The impact values of each of the variables are entered in the Java Program Applet based on the assumption that all the variables have a positive impact on each other.

\section{RESULTS}

After fixing the above relational matrix in the Fuzzy Cognitive Map applet as shown in Figure I, we start our iterations with different cases. Then, the important variables required to run the washing machine are identified. In each case ' $I$ ' stands for input values and ' $O$ ' stands for output values. The results got from the Fuzzy Cognitive Map applet have been shown from Table III to Table XII.

Figure I: Outlook of Fuzzy Cognitive Map applet

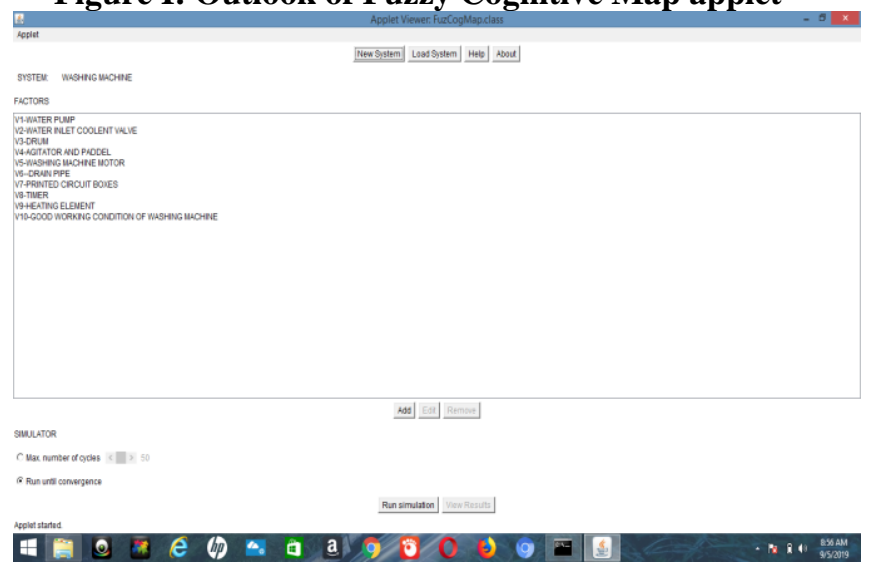




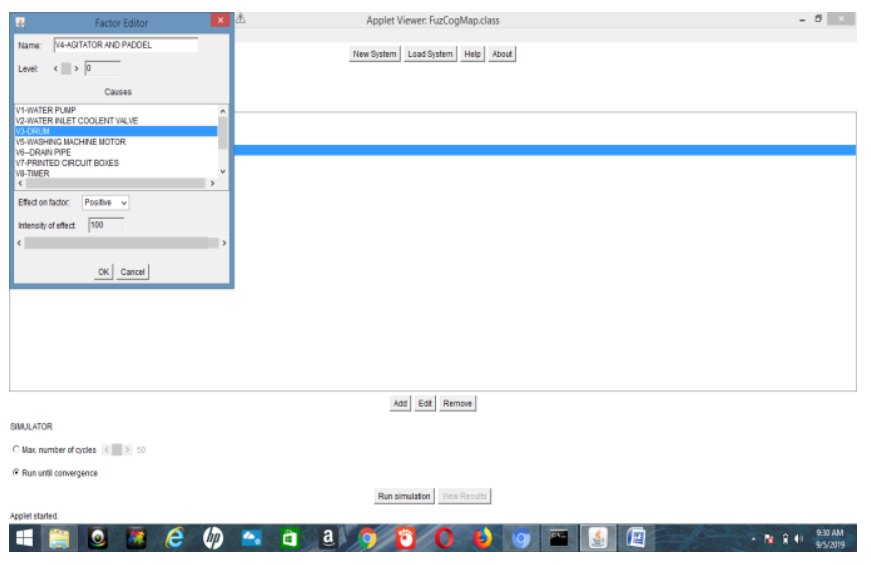

Table III: (12 iterations)

\begin{tabular}{|c|c|c|c|c|c|c|c|c|c|c|}
\hline & $\mathbf{V}_{1}$ & $\mathbf{V}_{2}$ & $\mathbf{V}_{3}$ & $\mathbf{V}_{4}$ & $\mathbf{V}_{5}$ & $\mathbf{V}_{6}$ & $\mathbf{V}_{7}$ & $\mathbf{V}_{8}$ & $\mathbf{V}_{9}$ & $\mathbf{V}_{10}$ \\
\hline $\mathrm{I}$ & $\mathbf{1 0 0}$ & $\mathbf{4 0}$ & $\mathbf{4 0}$ & $\mathbf{4 0}$ & $\mathbf{4 0}$ & $\mathbf{4 0}$ & $\mathbf{4 0}$ & $\mathbf{4 0}$ & $\mathbf{4 0}$ & $\mathbf{4 0}$ \\
\hline $\mathrm{O}$ & $\mathbf{4 2}$ & $\mathbf{4 2}$ & $\mathbf{4 2}$ & $\mathbf{4 1}$ & $\mathbf{4 1}$ & $\mathbf{4 2}$ & $\mathbf{4 1}$ & $\mathbf{4 1}$ & $\mathbf{4 2}$ & $\mathbf{4 0}$ \\
\hline
\end{tabular}

Table IV: (9 iterations)

\begin{tabular}{|c|c|c|c|c|c|c|c|c|c|c|}
\hline & $\mathbf{V}_{1}$ & $\mathbf{V}_{2}$ & $\mathbf{V}_{3}$ & $\mathbf{V}_{4}$ & $\mathbf{V}_{5}$ & $\mathbf{V}_{6}$ & $\mathbf{V}_{7}$ & $\mathbf{V}_{8}$ & $V_{9}$ & $\mathbf{V}_{10}$ \\
\hline $\mathrm{I}$ & $\mathbf{4 0}$ & $\mathbf{1 0 0}$ & $\mathbf{4 0}$ & $\mathbf{4 0}$ & $\mathbf{4 0}$ & $\mathbf{4 0}$ & $\mathbf{4 0}$ & $\mathbf{4 0}$ & $\mathbf{4 0}$ & $\mathbf{4 0}$ \\
\hline $\mathrm{O}$ & $\mathbf{4 2}$ & $\mathbf{4 2}$ & $\mathbf{4 2}$ & $\mathbf{4 1}$ & $\mathbf{4 1}$ & $\mathbf{4 2}$ & $\mathbf{4 1}$ & $\mathbf{4 1}$ & $\mathbf{4 2}$ & $\mathbf{4 0}$ \\
\hline
\end{tabular}

Table V: (17 iterations)

\begin{tabular}{|c|c|c|c|c|c|c|c|c|c|c|}
\hline & $\mathrm{V}_{1}$ & $\mathbf{V}_{2}$ & $\mathbf{V}_{3}$ & $\mathbf{V}_{4}$ & $\mathbf{V}_{5}$ & $\mathbf{V}_{6}$ & $\mathbf{V}_{7}$ & $\mathbf{V}_{\mathbf{8}}$ & $\mathrm{V}_{9}$ & $\mathrm{~V}_{10}$ \\
\hline $\mathrm{I}$ & $\mathbf{4 0}$ & $\mathbf{4 0}$ & $\mathbf{1 0 0}$ & $\mathbf{4 0}$ & $\mathbf{4 0}$ & $\mathbf{4 0}$ & $\mathbf{4 0}$ & $\mathbf{4 0}$ & $\mathbf{4 0}$ & $\mathbf{4 0}$ \\
\hline $\mathrm{O}$ & $\mathbf{4 2}$ & $\mathbf{4 2}$ & $\mathbf{4 2}$ & $\mathbf{4 1}$ & $\mathbf{4 1}$ & $\mathbf{4 2}$ & $\mathbf{4 1}$ & $\mathbf{4 1}$ & $\mathbf{4 2}$ & $\mathbf{4 0}$ \\
\hline
\end{tabular}

Table VI: (9 iterations)

\begin{tabular}{|c|c|c|c|c|c|c|c|c|c|c|}
\hline & $\mathrm{V}_{1}$ & $\mathbf{V}_{2}$ & $\mathbf{V}_{3}$ & $\mathbf{V}_{4}$ & $\mathbf{V}_{5}$ & $\mathbf{V}_{6}$ & $\mathbf{V}_{7}$ & $\mathbf{V}_{8}$ & $\mathbf{V}_{9}$ & $\mathrm{~V}_{10}$ \\
\hline $\mathrm{I}$ & $\mathbf{4 0}$ & $\mathbf{4 0}$ & $\mathbf{4 0}$ & $\mathbf{1 0 0}$ & $\mathbf{4 0}$ & $\mathbf{4 0}$ & $\mathbf{4 0}$ & $\mathbf{4 0}$ & $\mathbf{4 0}$ & $\mathbf{4 0}$ \\
\hline $\mathrm{O}$ & $\mathbf{4 1}$ & $\mathbf{4 1}$ & $\mathbf{4 1}$ & $\mathbf{4 1}$ & $\mathbf{4 1}$ & $\mathbf{4 1}$ & $\mathbf{4 1}$ & $\mathbf{4 1}$ & $\mathbf{4 1}$ & $\mathbf{4 0}$ \\
\hline
\end{tabular}

Table VII: (9 iterations)

\begin{tabular}{|c|c|c|c|c|c|c|c|c|c|c|}
\hline & $\mathbf{V}_{1}$ & $\mathbf{V}_{2}$ & $\mathbf{V}_{3}$ & $\mathbf{V}_{4}$ & $\mathbf{V}_{5}$ & $\mathbf{V}_{6}$ & $\mathbf{V}_{7}$ & $\mathbf{V}_{8}$ & $\mathbf{V}_{9}$ & $\mathbf{V}_{10}$ \\
\hline $\mathrm{I}$ & $\mathbf{4 0}$ & $\mathbf{4 0}$ & $\mathbf{4 0}$ & $\mathbf{4 0}$ & $\mathbf{1 0 0}$ & $\mathbf{4 0}$ & $\mathbf{4 0}$ & $\mathbf{4 0}$ & $\mathbf{4 0}$ & $\mathbf{4 0}$ \\
\hline $\mathrm{O}$ & $\mathbf{4 2}$ & $\mathbf{4 2}$ & $\mathbf{4 2}$ & $\mathbf{4 1}$ & $\mathbf{4 1}$ & $\mathbf{4 2}$ & $\mathbf{4 1}$ & $\mathbf{4 1}$ & $\mathbf{4 2}$ & $\mathbf{4 0}$ \\
\hline
\end{tabular}

Table VIII: (6 iterations)

\begin{tabular}{|c|c|c|c|c|c|c|c|c|c|c|}
\hline & $\mathbf{V}_{\mathbf{1}}$ & $\mathbf{V}_{2}$ & $\mathbf{V}_{3}$ & $\mathbf{V}_{4}$ & $\mathbf{V}_{5}$ & $\mathbf{V}_{6}$ & $\mathbf{V}_{7}$ & $\mathbf{V}_{8}$ & $\mathbf{V}_{9}$ & $\mathbf{V}_{10}$ \\
\hline $\mathrm{I}$ & $\mathbf{4 0}$ & $\mathbf{4 0}$ & $\mathbf{4 0}$ & $\mathbf{4 0}$ & $\mathbf{4 0}$ & $\mathbf{1 0 0}$ & $\mathbf{4 0}$ & $\mathbf{4 0}$ & $\mathbf{4 0}$ & $\mathbf{4 0}$ \\
\hline $\mathrm{O}$ & $\mathbf{4 2}$ & $\mathbf{4 2}$ & $\mathbf{4 2}$ & $\mathbf{4 1}$ & $\mathbf{4 1}$ & $\mathbf{4 2}$ & $\mathbf{4 0}$ & $\mathbf{4 0}$ & $\mathbf{4 1}$ & $\mathbf{4 0}$ \\
\hline
\end{tabular}

Table IX: (8 iterations)

\begin{tabular}{|c|c|c|c|c|c|c|c|c|c|c|}
\hline & $\mathrm{V}_{\mathbf{1}}$ & $\mathbf{V}_{2}$ & $\mathbf{V}_{3}$ & $\mathbf{V}_{\mathbf{4}}$ & $\mathbf{V}_{5}$ & $\mathbf{V}_{6}$ & $\mathbf{V}_{7}$ & $\mathbf{V}_{\mathbf{8}}$ & $\mathrm{V}_{9}$ & $\mathrm{~V}_{\mathbf{1 0}}$ \\
\hline $\mathrm{I}$ & $\mathbf{4 0}$ & $\mathbf{4 0}$ & $\mathbf{4 0}$ & $\mathbf{4 0}$ & $\mathbf{4 0}$ & $\mathbf{4 0}$ & $\mathbf{1 0 0}$ & $\mathbf{4 0}$ & $\mathbf{4 0}$ & $\mathbf{4 0}$ \\
\hline $\mathrm{O}$ & $\mathbf{4 0}$ & $\mathbf{4 0}$ & $\mathbf{4 0}$ & $\mathbf{4 0}$ & $\mathbf{4 0}$ & $\mathbf{4 0}$ & $\mathbf{4 0}$ & $\mathbf{4 0}$ & $\mathbf{4 0}$ & $\mathbf{4 0}$ \\
\hline
\end{tabular}

Table X (9 iterations):

\begin{tabular}{|c|c|c|c|c|c|c|c|c|c|c|}
\hline & $\mathbf{V}_{1}$ & $\mathbf{V}_{2}$ & $\mathbf{V}_{3}$ & $\mathbf{V}_{4}$ & $\mathbf{V}_{5}$ & $\mathbf{V}_{6}$ & $\mathbf{V}_{7}$ & $\mathbf{V}_{8}$ & $\mathbf{V}_{9}$ & $V_{10}$ \\
\hline $\mathrm{I}$ & $\mathbf{4 0}$ & $\mathbf{4 0}$ & $\mathbf{4 0}$ & $\mathbf{4 0}$ & $\mathbf{4 0}$ & $\mathbf{4 0}$ & $\mathbf{4 0}$ & $\mathbf{1 0 0}$ & $\mathbf{4 0}$ & $\mathbf{4 0}$ \\
\hline $\mathrm{O}$ & $\mathbf{4 0}$ & $\mathbf{4 0}$ & $\mathbf{4 0}$ & $\mathbf{4 0}$ & $\mathbf{4 0}$ & $\mathbf{4 0}$ & $\mathbf{4 0}$ & $\mathbf{4 0}$ & $\mathbf{4 0}$ & $\mathbf{4 0}$ \\
\hline
\end{tabular}

Table XII (12 iterations):

\begin{tabular}{|c|c|c|c|c|c|c|c|c|c|c|}
\hline & $\mathrm{V}_{1}$ & $\mathrm{~V}_{2}$ & $\mathbf{V}_{3}$ & $\mathbf{V}_{4}$ & $\mathrm{~V}_{5}$ & $\mathrm{~V}_{6}$ & $\mathrm{~V}_{7}$ & $\mathbf{V}_{8}$ & $\mathrm{~V}_{9}$ & $\mathrm{~V}_{10}$ \\
\hline $\mathrm{I}$ & $\mathbf{4 0}$ & $\mathbf{4 0}$ & $\mathbf{4 0}$ & $\mathbf{4 0}$ & $\mathbf{4 0}$ & $\mathbf{4 0}$ & $\mathbf{4 0}$ & $\mathbf{4 0}$ & $\mathbf{1 0 0}$ & $\mathbf{4 0}$ \\
\hline $\mathrm{O}$ & $\mathbf{4 2}$ & $\mathbf{4 2}$ & $\mathbf{4 2}$ & $\mathbf{4 1}$ & $\mathbf{4 1}$ & $\mathbf{4 2}$ & $\mathbf{4 1}$ & $\mathbf{4 1}$ & $\mathbf{4 2}$ & $\mathbf{4 0}$ \\
\hline
\end{tabular}

By using the above technique, it has been identified that all the parts in the washing machine plays an equal role.

\section{CIRCUIT CREATION FOR THE IDENTIFIED VARIABLES}

Since every variable has a major role in the working of washing machine, a circuit is created for all the nine variables.

First, a sensor is fixed for each part of the washing machine which is not in a proper working condition. Second, we assign either the value ' 0 ' or ' 1 '. Then, a constraint is fixed so that, the sensor will beep, if more than two parts are not working properly. Using this, a circuit with 46 minterms can be constructed. Now, in order to minimize the circuit, we use the software .

$$
\leftarrow \text { Boolean Function }
$$

Distinct Minimized Equation from Unique Boolean Function in Simple Truth Table Logic

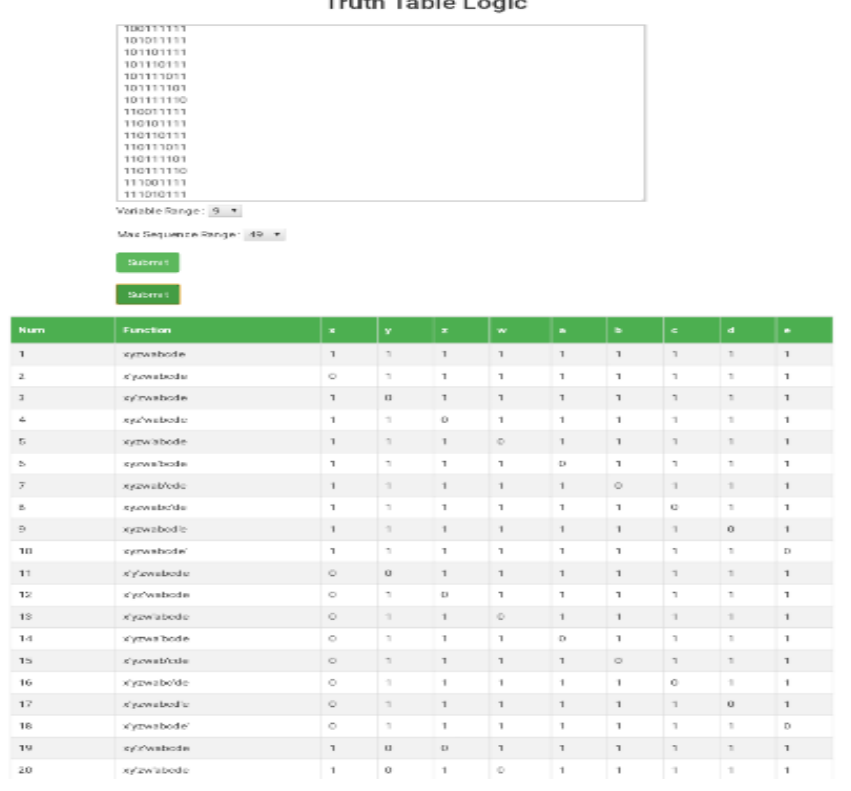



the cost in the Creation of a Circuit

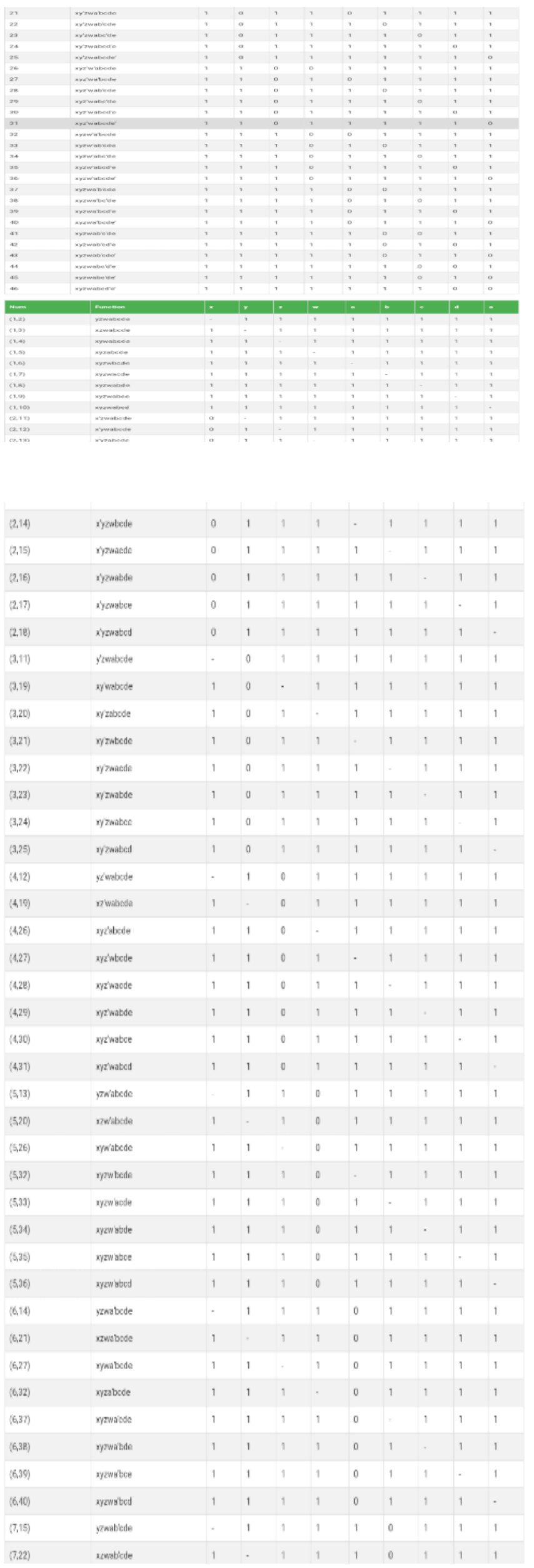

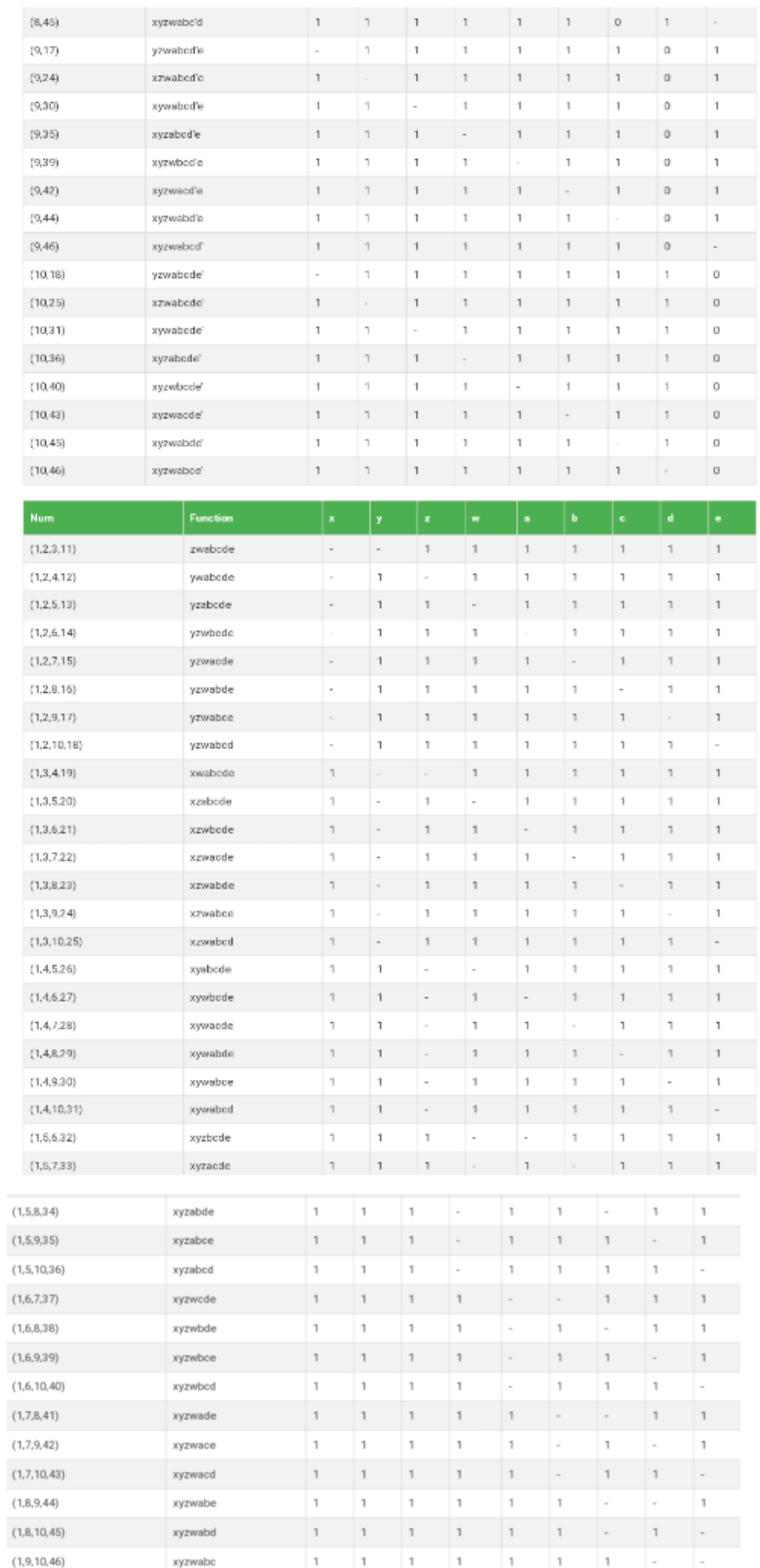

Figure II: Execution of the Result

Thus, the above Boolean function is reduced to a function having 36 minterms, thereby minimizing the cost of the circuit. The above can be accessed by the software https://s3.amazonaws.com/kanchanaboolean/index.html.

\section{APPLICATION}

We can use the above concept in several applications, especially in the electrical field. It reduces the cost of repairing the equipment as the sensors help us to identify the fault immediately.

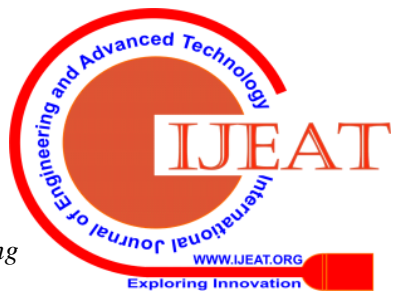


We have chosen the application as a washing machine because it is used as a home appliance in almost all the houses especially in the home of working professionals.

\section{CONCLUSION}

Using the Fuzzy Cognitive Map, the variables in the Boolean function has been reduced, thus arriving at the conclusion that, all the parts in the washing machine play a significant role. To reduce the cost of the circuit, the number of minterms was minimized from 46 to 36 using the Java scripts software. Hence, any fault in the equipment could be identified easily, before the machine gets totally damaged, using the sensors in the circuit.

\section{REFERENCES}

1 .Shannon C.E. (1938) A Symbolic analysis of relay and switching circuits.-Trans. AIEE.

2. Visvam Devadoss Ambeth Kumar and S. Gokul Amuthan "static structure simplification of Boolean function for ' $N$ ' variables - A novel approach", Journal of Microelectronics, Volume 01, Issue 4(2016), PP: 160-167

3. Sebestian P. Tomaszewski, Ilgaz U. Celik, George E. Antoniou, "WWW- Base Boolean Function Minimization", International journal of applied Mathematics and Computer science, Volume 13,Issue 4(2003), PP:577-583.

4. Kanchana A., Srinivasa Rao .K, "Indentifying the problem in Motorcycle using Boolean function" ,International Journal of Information Research and Review, Volume 03, Issue 11(2016), PP:3160-3167.

5. Kanchana A., Srinivasa Rao .K : "Reducing the Variables in Boolean Function Using Fuzzy Cognitive Map to Create a Circuit", International Journal of Pure and Applied Mathematics, Volume 119 Issue 9(2018)

6. Guillermo Ochoa de Aspuru, "java applet software for FuzzyCognitiveMaps",www.ochoadeaspuru.com/fuzcogmap/index. php.

7. Washing Machine, from Wikipedia, the free encyclopedia.

8. Kanchana A., Srinivasa Rao .K : "Software Approach to Minimize Boolean Function as 'n' Distinct Functions", Jour of Adv Research in Dynamical \& Control Systems, Volume 10 Issue 7(2018).

\section{AUTHORS PROFIL}

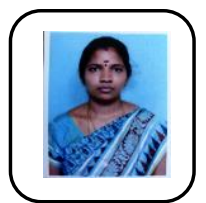

Dr.A.Kanchana,Ph.D, Assistant Professor, Departmen of Mathematics(H\&S), Rajalakshmi Institute of Technology, Kuthambakkam, Chennai-600 0124,Tamil Nadu.

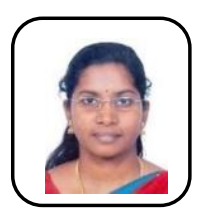

Ms.Vinitha Navis Varuvel, M.Sc, M.Phil Assistant Professor, Department of Mathematics(H\&S), Rajalakshmi Institute of Technology, Kuthambakkam, Chennai-600 0124,Tamil Nadu.

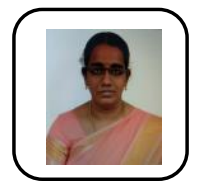

Ms.D. Samundeeswari, M.Sc, M.Phil ,( Ph. D) Assistant Professor(SS), Department of Mathematics(H\&S), Rajalakshmi Institute of Technology, Kuthambakkam, Chennai-600 0124,Tamil Nadu

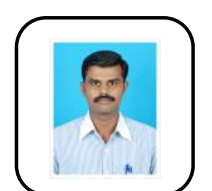

Mr.S. Kuppuraj, M.Sc, M.Phil ,Assistant Professor(SS) Department of Mathematics(H\&S), Rajalakshmi Institute of Technology, Kuthambakkam, Chennai-600 0124,Tamil Nadu

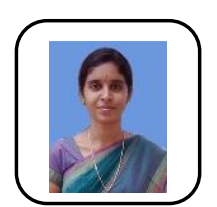

Ms.R. Kiruthika, M.Sc, M.Phil ,Assistant Professor, Department of Mathematics(H\&S), Rajalakshmi Institute of Technology, Kuthambakkam, Chennai600 0124,Tamil Nadu 